Recepción: 22/01/2019

Aceptación: 29/03/2019

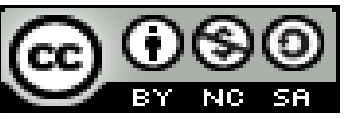

Ciencias técnicas y aplicadas

Publicación: 05/04/2019

Artículo de Instigación

\title{
Evaluación de la gestión de riesgos naturales en el sector hotelero de la cuidad de Manta
}

\section{Evaluation and validation of a sensor for counting and classification of polluting particles in lubricating oils: Case Universidad Politécnica de Valencia}

\section{Avaliação da gestão de riscos naturais no setor hoteleiro da cidade de Manta \\ Cesar Augusto Arias-Mendoza ${ }^{\mathrm{I}}$ arias.uleam@hotmail.com \\ Jouber Antonio Azua-Alvia II jouber.azua@uleam.edu.ec}

Correspondencia: arias.uleam@hotmail.com

${ }^{\text {I }}$ Magíster en Seguridad Salud y Ambiente Laboral, Ingeniero Industrial, Docente de la Universidad Laica Eloy Alfaro de Manabí. Manta. Ecuador.

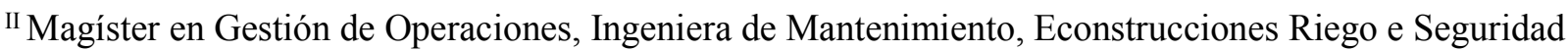
Industrial Seprytsa S.A., Docente de la Universidad Laica Eloy Alfaro de Manabí. Manta. Ecuador. 


\title{
Resumen
}

El presente trabajo de investigación tiene como objetivo principal analizar la gestión de los riesgos naturales en el sector hotelero de la ciudad de Manta después del terremoto del 16 de abril de 2016. La investigación es de tipo descriptiva de campo, realizada en dos etapas, la primera se efectuó en mayo de 2016, en la que se determinó las afectaciones físicas en la infraestructura de los diferentes tipos de alojamiento hotelero a través de una encuesta aplicada a 140 hoteles; la segunda etapa se realizó en octubre de 2018 con la finalidad de establecer mediante la técnica de la entrevista, la gestión que realiza el sector hotelero con relación a los riesgos naturales. Se encontró que un 62,7\% de los hoteles de la ciudad de Manta gestiona sus riesgos, y que los indicadores de la gestión de los riegos naturales no superan el $80 \%$, lo que resulta ser no satisfactorio. Como conclusión puede afirmarse que el conglomerado de hoteles no lleva a cabo una gestión de riesgos naturales acorde con la realidad geográfica de la ciudad de Manta.

Palabras Clave: Gestión; hoteles; terremoto; tsunami; riesgos.

\begin{abstract}
The main objective of this research work is to analyze the management of natural hazards in the hotel sector of the city of Manta after the earthquake of April 16, 2016. The investigation is of descriptive type of field, carried out in two stages, the first one was carried out in May of 2016, in which the physical affectations in the infrastructure of the different types of hotel accommodation were determined through a survey applied to 140 Hotels The second stage was carried out in October 2018 with the purpose of establishing by means of the technique of the interview, the management that makes the hotel sector with relation to the natural risks. It was found that $62.7 \%$ of the hotels in the city of Manta manage their risks, and that the indicators of the management of natural irrigations do not exceed $80 \%$, which proves to be unsatisfactory. As a conclusion it can be said that the comglomerado of hotels does not carry out a natural risk management chord.
\end{abstract}

Key Words: Management; hotels; earthquake; tsunami; risks.

\section{Resumo}

O principal objetivo deste trabalho de pesquisa é analisar a gestão de riscos naturais no setor hoteleiro da cidade de Manta após o terremoto de 16 de abril de 2016. A pesquisa é do tipo 
descritivo de campo, realizada em duas etapas, a em primeiro lugar, foi realizado em maio de 2016, no qual os efeitos físicos na infraestrutura dos diferentes tipos de alojamento em hotel foram determinados através de um inquérito aplicado a 140 hotéis; A segunda etapa foi realizada em outubro de 2018 com o objetivo de estabelecer, por meio da técnica da entrevista, a gestão realizada pelo setor hoteleiro em relação aos riscos naturais. Verificou-se que $62,7 \%$ dos hotéis da cidade de Manta gerenciam seus riscos, e que os indicadores de gestão dos riscos naturais não ultrapassam 80\%, o que acaba por ser insatisfatório. Em conclusão, pode-se afirmar que o conglomerado de hotéis não realiza uma gestão de risco natural de acordo com a realidade geográfica da cidade de Manta.

Palavras-chave: Gestão; hotéis; terremoto tsunami; riscos.

\section{Introducción}

Resulta claro que cuando se quiere mejorar algún proceso, este tiene que ser gestionado (Lavell, 1999), de allí la importancia de gestionar los riesgos naturales en el sector hotelero de la cuidad de Manta, partiendo del hecho de la ocurrencia de desastres naturales del pasado reciente, tanto en Ecuador como en distintas partes del mundo, obligando de tal forma a mandatarios y empresarios del sector hotelero a trabajar en la prevención y protección de los recursos humanos y materiales a fin de mitigar los desastres predecibles y no predecibles.

Los desastres están clasificado según Ventura-Velázquez et al.; (2009), en naturales y tecnológicos, los desastres naturales están clasificados por los procesos dinámicos que se producen al interior de la tierra, los que se llevan a cabo en la superficie, los meteorológicos y los de origen biológico, es evidente que los desastres naturales que se producen al interior de la tierra como sismos, tsunamis y erupciones volcánicas son las que mayormente han azotado a las cuatro regiones del Ecuador (Bustos, 2010), sorprendiendo al mundo que en un país tan pequeño tenga un alto número de volcanes varios de ellos activos y que sus costas haya sido afectado por terremotos y tsunami de gran magnitud como el terremoto que azotó las costas de la frontera entre Ecuador y Colombia en el año 1906, considerado uno de los terremotos más grande de la historia reciente, que vino acompañado de un tsunami que desbasto las costas de nuestros 
hermanos Esmeraldeños siendo tomado este evento como referente por lo que causo en el suelo marino.

Manta al ubicarse en el perfil costero ecuatoriano ha sido afectada considerablemente en el último 74 años por cuatro sismos en intensidades mayor a VII grados (escala de Mercalli modificada) tomando la recurrencia como referencia para estar preparado más que todo en el campo hotelero donde se alojan según su capacidad grandes cantidades de personas, los niveles de prevención y protección deben ser más alto que para cualquier otra actividad, y sus infraestructuras deben garantizar la resistencia frente a los desastres naturales, tecnológicos y antrópicos, a fin de minimizar el impacto que dejó el terremoto del 16 de abril del 2016, evento que causó grandes pérdidas, entre ellas un total de 209 fallecidos e innumerables daños materiales en la ciudad de Manta siendo el sector hotelero uno de los más afectados (Corral-Muñoz, 2018).

Con estos antecedentes la presente investigación plantea dos objetivos, el primero determinar las diferentes afectaciones físicas en la estructura de la planta hotelera para cuantificar las pérdidas que dejó el evento de 16 de abril y el segundo analizar la gestión de los riesgos naturales en el sector hotelero para determinar las medidas y acciones ejecutadas post-desastre, y de esta manera conocer si la gestión que está realizando el sector hotelero es satisfactoria o no satisfactoria.

El presente trabajo ha tomado como fuentes bibliográficas varios estudios que abordan los desastres pasados en el mundo, en América y en el Ecuador (Caputo, et al., 1985; Basabe et al., 1996; Lugo Hubp \& Inbar, 2002). Por ejemplo, a nivel local, se consultó un estudio de la Municipalidad de Manta (2010) el cual determina la alta amenaza sísmica para el Cantón.

\section{Importancia del Problema}

El Ecuador al ubicarse en el cinturón de fuego (Geo Enciclopedia, 2017) (Figura $\mathrm{N}^{\circ}$ 1), no solo es tierra de volcanes (Mothes, 1998), ya que a más de su elevada actividad volcánica su perfil costero se asienta sobre una zona de alta actividad tectónica, que constantemente acumula energía, que al ser liberada origina movimientos telúricos y en determinados casos, tsunamis. Fue así como esta energía liberada causó el terremoto del 16 de abril del 2016, donde las provincias de Manabí y Esmeradas fueron las más afectadas, alcanzando la mayor cantidad de víctimas, desaparecidos y pérdidas materiales: ¿Porque estas lamentables pérdidas? fue la pregunta de algunos familiares de las víctimas cuando paso la tragedia: Uno de los problemas es 
el cortoplacismo de nuestra población para actuar frente a cualquier evento y luego olvidarse de lo ocurrido, no se tiene memoria histórica de la recurrencia de los desastres naturales y una gran parte del colectivo público y privado no estuvo preparado para este trágico evento.

Las costas ecuatorianas están llenas de nefastas experiencias por los movimientos telúricos y tsunamis registrados a lo largo de la historia (López, 2013), desde el año 1942 al 2016, promediando una recurrencia entre evento de aproximadamente entre 10 a 15 años, con intensidades entre VII y IX grados (escala de Mercalli modificada), enfatizando que este análisis estadístico puede ser utilizado como una herramienta mas no como una regla para determinar el riesgo, relacionando la combinación de la probabilidad o amenaza con la vulnerabilidad de los elementos expuestos. Por lo anterior y por la ubicación geográfica de Manta frente a las costas del Pacifico. se estima un riesgo alto ante la ocurrencia de movimientos sísmicos de mediana y gran intensidad.

\section{Metodología}

Hernández Sampieri et al., (2014), sostienen que la selección del diseño metodológico de cualquier investigación, puede se experimental o no experimental. Los diseños no experimentales permiten observar los fenómenos tal como se dan en su contexto natural para posteriormente analizarla clasificándose en: Longitudinal y Transeccional o transversal.

Agregan Hernández-Sampieri et al., (2014), que las investigaciones no experimentales longitudinales hacen mención a estudios que obtienen datos de grupos en diferentes puntos del tiempo para realizar una conclusión acerca de la evolución de las causas y sus efectos, dividiéndose en: Diseños de Tendencia, Diseños Evolutivos de grupos y Diseño panel; mientras que las investigaciones transeccionales o transversales son las que recopilan datos en un momento único. Este tipo de diseño puede dividirse en exploratorio, Descriptivos y Correlaciónal-Causal (Ander-Egg, 2010; Hernández-Sampieri, et al., 2012).

Acogiendo los criterios de una investigación no experimental transeccional o transversal, se evaluó la gestión de los riesgos naturales en las empresas hoteleras de Manta, recolectando datos pertinentes, lo que permitió posteriormente analizarlos para poder determinar las afectaciones en los establecimientos del sector hotelero. 

Con los criterios de una investigación no experimental transeccional o transversal descriptiva se describieron los tipos de alojamiento, las afectaciones post desastre, la ubicación geográfica y el número de personas que quedaron sin empleo; adicionalmente se describieron datos generales relacionados con planificación de acciones ante la posible ocurrencia de eventos naturales con capacidad destructiva, tal es el caso de los terremotos.

\section{Resultados}

Manabí es una de las cinco provincias ubicadas en el perfil costero ecuatoriano frente al océano Pacifico que ha sido testigos de varios terremotos de altas intensidades de acuerdo a datos históricos que toman como referencia el mega-terremoto de magnitud 8.3 (Manchuel, et al., 2009), que pasó entre la frontera de Ecuador y Colombia, (Estrada \& Farbiarz, 2005), devastando la costa de Esmeraldas en el año 1906 y causando una ruptura en el lecho marino de 400 a 500 km de longitud, ubicándose en la zona de interface de subducción, a 90 kilómetros al Noreste del terremoto del 16 de abril de 2016. Los terremotos posteriores al mega-terremoto de año 1906 registrados en la provincia de Manabí (Tabla $\mathrm{N}^{\circ} 1$ ) se ubican dentro de la zona de ruptura alrededor de los 250 kilómetros y los diferentes epicentros de los terremotos desde el año 1942 hasta el de 2016 han formado una figura elíptica natural

Tabla $\mathbf{N}^{\circ}$ 1. Terremotos registrados en los últimos 74 años en la Provincia de Manabí

\begin{tabular}{|c|c|c|c|c|}
\hline $\mathrm{N}^{\circ}$ & Fecha & Intensidad & Zona & Efectos \\
\hline 1 & 31 enero 1906 & IX & $\begin{array}{l}\text { Esmeraldas- } \\
\text { Nariño }\end{array}$ & $\begin{array}{l}\text { Generó un tsunami de grandes } \\
\text { proporciones. }\end{array}$ \\
\hline 2 & 14 mayo 1942 & IX & $\begin{array}{l}\text { Manabí- } \\
\text { guayas }\end{array}$ & $\begin{array}{l}\text { Daños en Manabí, Guayas, Los Ríos, } \\
\text { Esmeraldas, Bolívar e Imbabura }\end{array}$ \\
\hline 3 & 19 mayo 1964 & VII & Manabí & $\begin{array}{l}\text { Fuerte terremoto con epicentro en la } \\
\text { provincia de Manabí, donde varias } \\
\text { poblaciones resultaron muy afectadas. }\end{array}$ \\
\hline 4 & $\begin{array}{ll}04 & \text { agosto } \\
1998 & \end{array}$ & VII & Manabí & $\begin{array}{l}\text { Terremoto de severas consecuencias en la } \\
\text { provincia de Manabí. Gran destrucción de } \\
\text { edificios en Bahía de Caráquez. Daños } \\
\text { graves en Canoa, San Vicente y localidades } \\
\text { cercanas. }\end{array}$ \\
\hline 5 & 16 abril 2016 & IX & $\begin{array}{l}\text { Manabí/ } \\
\text { pedernales }\end{array}$ & $\begin{array}{l}\text { Terremoto de severas consecuencias } \\
\text { Manabí, Esmeraldas y otras provincias de } \\
\text { la costa ecuatoriana, ubicación e intensidad } \\
\text { similar al terremoto del año } 1942 \text {. }\end{array}$ \\
\hline
\end{tabular}

Fuente: Basado en López (2013). 
Manta con una extensión de $309 \mathrm{Km}^{2}$ y 217.600 habitantes (INEC, 2010), fue declarada en el año 2009 como Puerto Turístico del Ecuador por el Ministerio de Turismo, y para todos los ecuatorianos es un icono turístico visitada por cientos de turistas nacionales y extranjeros, destacando la amabilidad de su gente y las bondades de su naturaleza, ofreciéndole a los turistas varias alternativas de diversión y distracción. Manta para los Manabitas es el eje económico de la provincia, razón por la cual el sector hotelero de la ciudad debe garantizar la seguridad de los usuarios y de su personal de planta frente a cualquier amenaza propias del sector ya sea de origen geológica (sismos, maremotos o tsunamis y deslizamientos), o hidrológicas (inundaciones).

El evento sísmico del 16 de abril de 2016 marca un antes y un después en la ciudadanía Mantense, pues nunca había vivido una eventualidad natural de la magnitud registrada (7,8 escala Richter), con lecciones aprendidas por las consecuencias que el mismo dejó. Este evento será recordado por muchos años, algunos por la pérdida de la vida de familiares y otros por las pérdidas materiales de muchas edificaciones que colapsaron totalmente, donde el esfuerzo de trabajo de varios años quedó destruido en apenas 58 segundos, y aun después de dos años, muchos no han podido reconstruir sus viviendas y negocios. Los apoyos económicos para la reconstrucción por la parte pública y privada no han llegado al 100\% de los damnificados, siendo la parroquia Tarqui la zona más afectada, donde hay en la actualidad varios lotes de terrenos baldíos por la demolición de las viviendas afectadas por el sismo.

Como resultado de la investigación se identificaron cuatro sectores de distintos niveles de afectación:

- Sector $\mathrm{N}^{\circ} 1$ Centro de la parroquia Manta ubicado a una altitud comprendida entre los 6 y 120 m.s.n.m. dividido en Centro, Centro Norte y Centro Sur hasta la parroquia San Mateo;

- Sector $\mathrm{N}^{\circ} 2$ Parroquias Tarqui, y Los Esteros, ubicado a una altitud comprendida entre los 6 y 40 m.s.n.m., llegando hasta los límites con Jaramijó;

- Sector N³ Parroquia Eloy Alfaro o Norte de la ciudad de Manta, ubicado a una altitud comprendida entre los 40 y 120 m.s.n.m., hasta los límites con Montecristi y al Sector

- $\quad N^{\circ} 4$ le correspondió a la Zona Rural parroquias San Lorenzo y Santa Marianita ubicadas a una altitud comprendida entre los 6 y 400 m.s.n.m.

Datos de las afectaciones causadas por el sismo se puede apreciar en la tabla 2. 
Tabla $\mathbf{N}^{\circ}$ 2. Efectos del terremoto en distintos sectores de la ciudad de Manta

\begin{tabular}{llllll}
\hline \multicolumn{1}{c}{ Sector } & \multicolumn{1}{c}{$\begin{array}{c}\text { Colapso } \\
\text { total }\end{array}$} & $\begin{array}{c}\text { Afectación } \\
\text { parcial }\end{array}$ & $\begin{array}{c}\text { Ninguna } \\
\text { afectación }\end{array}$ & $\begin{array}{c}\text { Por } \\
\text { confirmar }\end{array}$ & Total \\
\hline Centro, Centro Sur y Centro Norte & 12 & 44 & 7 & 4 & 67 \\
Tarqui, Los esteros y Palmar & 24 & 12 & 8 & 4 & 48 \\
Norte & 0 & 7 & 5 & 3 & 15 \\
Zona rural & 0 & 4 & 4 & 2 & 10 \\
Total & 36 & 67 & 24 & 13 & 140 \\
\hline \multicolumn{5}{l}{ Fuente: Elaboración propia. }
\end{tabular}

En función del color de las etiquetas pegadas en los edificios inspeccionados por los técnicos del Comité de Operaciones Emergentes (COE) y el Ministro de Desarrollo Urbano y Vivienda (MIDUVI), en los diferentes hoteles (roja para las edificaciones colapsadas totalmente y parcialmente con orden de derrocamiento, amarilla con prohibiciones de habitabilidad y verde para las que no tenían ninguna restricción) y con la información de las encuestas realizadas se resumen las afectaciones post desastre de todos los hoteles de Manta (Tabla $N^{\circ} 3$ ).

Tabla $\mathbf{N}^{\circ}$ 3. Resumen del total de hoteles afectados en Panta por el terremoto de abril de 2016.

\begin{tabular}{lcc}
\hline \multicolumn{1}{c}{ Tipo de afectaciones } & $\mathrm{N}^{\circ}$ de Hoteles afectados & $\%$ \\
\hline Colapso total & 35 & $25 \%$ \\
Afectación parcial no operativo & 12 & $9 \%$ \\
Afectación parcial operativo & 69 & $49 \%$ \\
Ninguna & 24 & $17 \%$ \\
Total & 140 & $100 \%$ \\
\hline
\end{tabular}

Fuente: Elaboración propia.

Los hoteles colapsados totalmente y los afectados parcialmente fue la causa en la disminución de la capacidad de alojamiento resumido en la tabla $\mathrm{N}^{\circ} 4$. Pero además tuvo impacto negativo en el ámbito laboral, pues el cierre de algunos hoteles elevó el número de desempleados ( $\mathrm{Tabla}^{\circ}{ }^{\circ}$ ) 
Tabla $\mathrm{N}^{\circ} 4$. Números de habitaciones con sus respectivas afectaciones por el terremoto 16 A.

\begin{tabular}{lcc}
\hline \multicolumn{1}{c}{ Tipo de afectaciones } & $\mathbf{N}^{\circ}$ de Habitaciones afectadas & $\mathbf{\%}$ \\
\hline Colapso total & 375 & $18 \%$ \\
Afectación parcial & 1274 & $60 \%$ \\
Sin afectación & 366 & $17 \%$ \\
Por confirmar & 96 & $5 \%$ \\
TOTAL & 2111 & $100 \%$ \\
\hline
\end{tabular}

Fuente: Elaboración propia.

Tabla $\mathbf{N}^{\circ}$ 5. Total, personas desempleadas como consecuencia del sismo del $16 \mathrm{~A}$.

\begin{tabular}{llll}
\hline \multicolumn{1}{c}{ Tipo de afectaciones } & & Personas desempleadas & $\%$ \\
\hline Hoteles con afectaciones parciales & 98 & $68 \%$ \\
Hoteles colapsados totalmente & 47 & $32 \%$ \\
Total hoteles afectados & 145 & $100 \%$ \\
\hline
\end{tabular}

Fuente: Elaboración propia.

Otro de los resultados de la investigación tiene que ver con las acciones de atención inmediata de los organismos de primera respuesta, Cuerpo de Bomberos, Fuerza Pública, Cruz Roja y la Secretaria de Gestión de Riesgos. En ese sentido, manifestaron los entrevistados que todos esos entes redoblaron fuerzas en el rescate de las víctimas que se encontraban atrapadas en las estructuras colapsadas y en la atención a los dannificados. Con el apoyo posterior de Organismos Nacionales de las diferentes Municipalidades del País y de Organizaciones internacionales de ayuda humanitaria ONGs., ONU y otras organizaciones que trabajaron con el Comité de Operaciones Emergentes Cantonal (COE) en las diferentes operaciones de rescate y atención a los damnificados, y en el restablecimiento de los servicios básicos en un $75 \%$ de la población Mantense en aproximadamente 15 días.

La segunda etapa de esta investigación se realizó en octubre de 2018, a dos años y 6 meses del fatídico 16 de abril de 2016, con el firme propósito de analizar la gestión de los riesgos naturales en los establecimientos hoteleros de la ciudad de Manta, tomando en cuenta los factores relacionados con la seguridad de los usuarios, el personal de planta y su preparación para actuar, principalmente frente a los eventos relacionados con la geodinámica interna.

La presente etapa de la investigación tuvo como objetivo general determinar mediante la aplicación de una encuesta la gestión de los riesgos naturales que están realizando los empresarios en los establecimientos hoteleros de la ciudad; de un total de 140 hoteles registrados 
en la base de datos quedaron operativos 93, de los cuales 72 colaboraron voluntariamente respondiendo a la encuesta.

La primera pregunta pretende determinar cuántos establecimientos cuentan con un profesional en Seguridad y Salud en el Trabajo (Tabla $\mathrm{N}^{\circ} 6$ ) capacitado para gestionar bajo cualquier modalidad de asistencia técnica los riesgos laborales naturales, antrópicos y tecnológicos.

Tabla N$^{\circ}$ 6. Asistencia técnica en Sistemas de Seguridad del Trabajo (SST)

\begin{tabular}{lcc}
\hline \multicolumn{1}{c}{ Modalidad de asistencia técnica } & $\mathbf{N}^{\circ}$ de técnicos & $\mathbf{\%}$ \\
\hline Responsable en SST & 11 & $15 \%$ \\
Técnico en SST & 0 & $0 \%$ \\
Asesor (servicios profesionales) & 21 & $29 \%$ \\
Ninguno & 40 & $56 \%$ \\
\hline
\end{tabular}

Fuente: Elaboración propia.

En la tabla $\mathrm{N}^{\circ} 7$ se resumen las consideraciones generales mínimas que deben conocer todos los trabajadores que laboran para las empresas hoteleras, independientemente cual sea el tipo de alojamiento.

Tabla $\mathbf{N}^{\circ}$ 7. Consideraciones generales mínimas que deben conocer los trabajadores de las empresas hoteleras.

\begin{tabular}{lccc}
\hline Descriptores & N/C & NO & SI \\
\hline $\begin{array}{l}\text { La construcción es sismo resistente y se encuentra en buen estado } \\
\text { La empresa opera en un área geográfica o sector afectado por el evento } \\
\text { del 16 A }\end{array}$ & 4 & 6 & 62 \\
$\begin{array}{l}\text { Conoce las amenazas naturales que pueden afectar la empresa } \\
\begin{array}{l}\text { Conoce usted cual es el grado de vulnerabilidad en la fiabilidad de la } \\
\text { construcción }\end{array}\end{array}$ & 34 & 36 \\
$\begin{array}{l}\text { Conoce usted los organismos nacionales y/o locales de respuesta ante } \\
\text { desastres }\end{array}$ & 11 & 23 & 67 \\
$\begin{array}{l}\text { Conoce usted el Plan N de la Secretaria de Gestión de Riesgo } \\
\text { "Responde EC." }\end{array}$ & 0 & 41 & 31 \\
\hline
\end{tabular}

Fuente: Elaboración propia.

El éxito de todo sistema de gestión depende del compromiso en todas las funciones y los niveles de una organización bajo el liderazgo de la alta dirección pudiendo las organizaciones aprovechar las oportunidades de prevenir o mitigar un impacto o una amenaza que se quiera mejorar controlándola en las diferentes fases de un proceso (Banco Interamericano de Desarrollo, 2012), 
para el caso del presente estudio, permite gestionar las amenazas naturales que pueden afectar la seguridad física de sus ocupantes y la continuidad del negocio, durante o después de un evento natural, fundamentado en el ciclo de Edwards Deming, también conocido como círculo PHVA, modelo que es muy usados por las organizaciones que buscan la mejora continua, pudiendo describirse su aplicación en la gestión de los riesgos naturales en las empresas hoteleras de Manta, de la siguiente forma:

Planificar: la encuesta establece las asignaciones de tareas de acuerdo la preparación para las diferentes actividades, el entrenamiento y la capacitación para alcanzar el reconocimiento de las partes interesadas de la gestión de los riesgos naturales (Tabla $\mathrm{N}^{\circ} 8$ ).

Hacer: la encuesta establece la implementación de los procesos según lo planificado (Tabla $\mathrm{N}^{\circ}$ 9).

Verificar: hacer el seguimiento y medir los procesos, verificando lo implementado e informando sus resultados (Tabla $\left.\mathrm{N}^{\circ} 10\right)$.

Actuar: emprender acciones y actualizaciones para determinar la mejora continua (Tabla $\left.\mathrm{N}^{\circ} 11\right)$.

Tabla $\mathbf{N}^{\circ}$ 8. Gestión en la Planificación

\begin{tabular}{lccc}
\hline Contenidos de la planificación & N/C & NO & SI \\
\hline $\begin{array}{l}\text { Se mantiene un Reglamento de SST registrado donde se estime los riesgos } \\
\text { naturales }\end{array}$ & 4 & 28 & 40 \\
$\begin{array}{l}\text { Se mantiene un Plan de Autoprotección debidamente aprobado el Cuerpo de } \\
\text { Bomberos }\end{array}$ & 5 & 11 & 56 \\
$\begin{array}{l}\text { El plan ha definido los niveles de riesgo que puede afectar la continuidad del } \\
\text { negocio }\end{array}$ & 3 & 26 & 43 \\
$\begin{array}{l}\text { Se ha planificado ejercicios de simulaciones de evacuaciones para los } \\
\text { eventos naturales }\end{array}$ & 0 & 30 & 42 \\
$\begin{array}{l}\text { Se ha planificado con la municipalidad de Manta ejercicios de simulaciones } \\
\text { de tsunami }\end{array}$ & 2 & 38 & 32 \\
Se mantiene activas la plataforma de capacitación para eventos naturales & 8 & 22 & 42 \\
\hline \multicolumn{1}{c}{ Fuente: Elaboración propia. } & &
\end{tabular}


Tabla $\mathbf{N}^{\circ} 9$ Gestión en el Hacer.

\begin{tabular}{lccc}
\hline Contenidos del Hacer & N/C & NO & SI \\
\hline $\begin{array}{l}\text { El plan de autoprotección esta implementado y socializado } \\
\begin{array}{l}\text { Se mantiene señalizada claramente las rutas de evacuación las salidas de } \\
\text { emergencia }\end{array}\end{array}$ & 0 & 5 & 56 \\
$\begin{array}{l}\text { Se mantiene señalizado claramente y ubicado el punto de encuentro local. } \\
\text { La empresa cuenta con alarmas de alerta, articuladas con las alarmas }\end{array}$ & 0 & 15 & 57 \\
$\begin{array}{l}\text { tempranas contra tsunamis } \\
\text { Se han ejecutado ejercicios y simulaciones de evacuaciones para los } \\
\text { eventos naturales }\end{array}$ & 2 & 34 & 36 \\
$\begin{array}{l}\text { Se ha participado en los simulacros zonales organizados por la municipio } \\
\text { de Manta }\end{array}$ & 2 & 27 & 45 \\
\hline
\end{tabular}

Fuente: Elaboración propia.

Tabla $\mathbf{N}^{\circ}$ 10. Gestión en la Verificación

\begin{tabular}{lccc}
\hline Contenidos de la Verificación & N/C & NO & SI \\
\hline $\begin{array}{l}\text { La empresa cuenta con registros de los simulacros realizados } \\
\text { Se han verificado los tiempos en los ejercicios de simulaciones de } \\
\text { evacuaciones }\end{array}$ & 5 & 41 & 26 \\
$\begin{array}{l}\text { Se ha evaluado la salud física y psicológica de los brigadistas de } \\
\text { emergencias }\end{array}$ & 6 & 42 & 24 \\
\hline
\end{tabular}

Fuente: Elaboración propia.

Tabla No 11. Gestión en la Actuación

\begin{tabular}{|c|c|c|c|}
\hline Contenidos de la Actuación & $\mathbf{N} / \mathbf{C}$ & NO & SI \\
\hline $\begin{array}{l}\text { Se mantiene actualizaciones de las normativas e instrumentos legales } \\
\text { en los planes y procedimientos de emergencias relacionadas con la } \\
\text { respuesta ante eventos naturales }\end{array}$ & 5 & 25 & 42 \\
\hline
\end{tabular}

Fuente: Elaboración propia.

\section{Discusión}

Los eventos sísmicos de alta magnitud en la provincia de Manabí han tenido una recurrencia entre 10 y 15 años, promediando los cuatro terremotos de intensidad superior a los VII grados escala de Mercalli modificada (Tabla $\mathrm{N}^{\circ} 1$ ). Llama la atención dos de ellos, que ubican sus epicentros muy cerca uno del otro, los terremotos de 1942 y 2016 son comparables por su ubicación en la misma zona de ruptura, en la misma falla y en las intensidades de sus réplicas. Según Demoraes \& D'ercole, (2001) la ocurrencia temporal de este tipo de eventos se conoce como ciclo sísmico. 
Ambos terremotos dejaron atrás innumerables pérdidas, pero por la ubicación en el tiempo se puede estimar que el terremoto del año 2016 causo más pérdidas al sector hotelero de Manta respecto al de 1942, registrándose en el 2016 un total de 35 hoteles completamente colapsados y 12 con afectaciones parciales que un no están operativos, reduciendo la capacidad de operación en la empresa hotelera en un 18\% de las 375 habitaciones de los hoteles colapsados y en materia laboral 145 trabajadores quedaron desempleados.

Los terremotos y maremotos "tsunamis" son frecuentes cerca de los bordes de las placas tectónicas; sin embargo, en Manabí no se registran maremotos de la magnitud que se han registrado en otros países que forman el cinturón de fuego del Pacífico, pero la amenaza está presente para los hoteles que se encuentran cerca de las playas, principalmente para los sectores bajos de las parroquias Tarqui y Los esteros, que se ubican a una alttitud entre los 6 y 40 (m.s.n.m.) metros sobre el nivel del mar.

En Manabí, el Ministerio Coordinador de Seguridad, la Secretaria de Gestión de Riesgos y el Servicio Integrado de Seguridad ECU 911 en un supuesto que se pueda alertar a una población para que se ubique en sitios seguro, instalaron en 14 cantones de la provincia de Manabí (Figura $\mathrm{N}^{\circ}$ 2) el 15 de diciembre de 2016, un total de 56 sirenas que forman el Sistema de Alerta Temprana (SAT) para Tsunami y represas para la difusión de alertas masivas a la ciudadanía. En Manta particularmente se ha instalado un total de 12 sirenas, 7 están en el centro de Manta, Tarqui y los Esteros, 1 en Piedra Larga y el resto entre San Mateo, Santa Marianita, luego de esto se programó dos simulacros sin haber tenido la participación mayoritaria de la población Mantense.

Según se conoció con esta investigación, el terremoto del 16 de abril de 2016 dejó varias lecciones aprendidas (Alvarado, (2018), como edificaciones de baja calidad constructiva, deficiente prevención por parte de los empresarios hoteleros, muy poca información e inspección de los órganos públicos que controlan las edificaciones y la seguridad integral de los establecimientos. No obstante, existe preocupación por cuanto sólo el $56 \%$ de los hoteles de Manta cuenta con la asistencia de un responsable o técnico en Seguridad y Salud laboral.

Los resultados respecto a la gestión de los riesgos naturales en los establecimientos hoteleros de la ciudad permiten afirmar que a más de dos años del lamentable evento del 16 abril de 2016 no se trabaja en prevención en la mayor parte del sector, de tal forma que los porcentajes parciales y 
promediados están muy por debajo de lo aceptable, así, el 56\% de los hoteles no disponen de un profesional de Seguridad que gestione los riesgos propios de la actividad, este análisis evidentemente se refleja en los indicadores insatisfactorios de las cuatro etapas de la gestión de los riesgos naturales, promediando los porcentajes resultantes en la Planificación, el Hacer, el Verificar y el Actuar es del 76\%, considerándolo como insatisfactorio.

Los empresarios hoteleros deben reformular su compromiso con la prevención y protección frente a los desastres naturales, como estratégica para la sostenibilidad de la actividad turística en Manta.

\section{Conclusión}

El estado ecuatoriano en relación al Artículo 389 de la Constitución Política Ecuatoriana manifiesta que el Estado protegerá a las personas, las colectividades y la naturaleza frente a los efectos negativos de los desastres de origen natural o antrópico, mediante la prevención ante el riesgo. Los Organismos de Control deben socializar y llegar a todos los actores de las empresas hoteleras tanto de los sectores urbanos como rurales con las acciones interpuestas para minimizar los riesgos naturales.

Los índices parciales y totales en Manta, respecto al tema tratado, no superan el $80 \%$, por lo cual la gestión básica de los riesgos naturales es insatisfactoria, de allí que las empresas hoteleras deben invertir en la contratación de profesionales especialistas en seguridad del Trabajo, tales como técnicos o asesores, de tal forma que la gestión de riesgos desde distintas ópticas sea una actividad eficiente.

Las empresas hoteleras deben trabajar en armonía con las líneas de investigación que realiza la academia, pues de esa forma se puede determinar fortalezas, oportunidades, debilidades y amenazas ante la ocurrencia de eventos adversos. 


\section{Anexos}

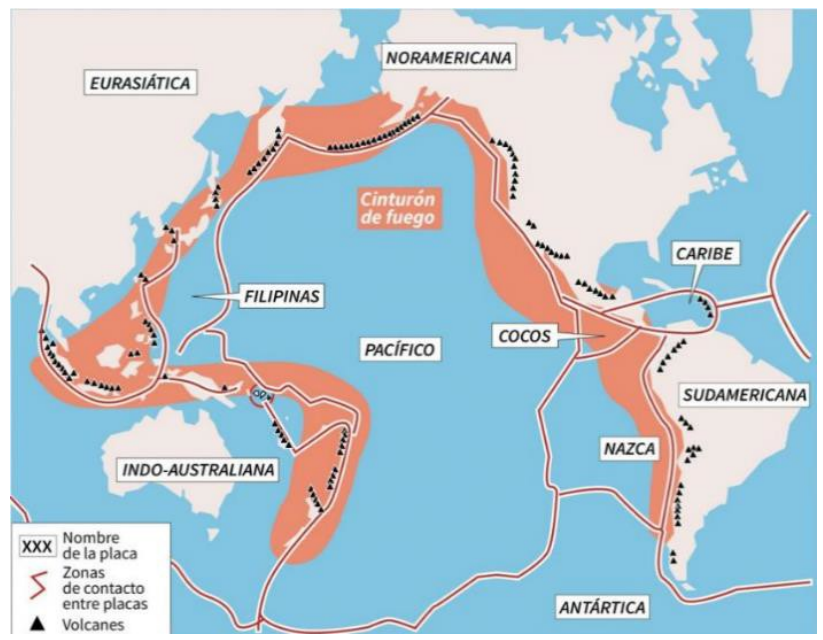

Figura 1. Mapa del Cinturón de fuego del Pacífico

Fuente: Geo Enciclopedia, (2017)

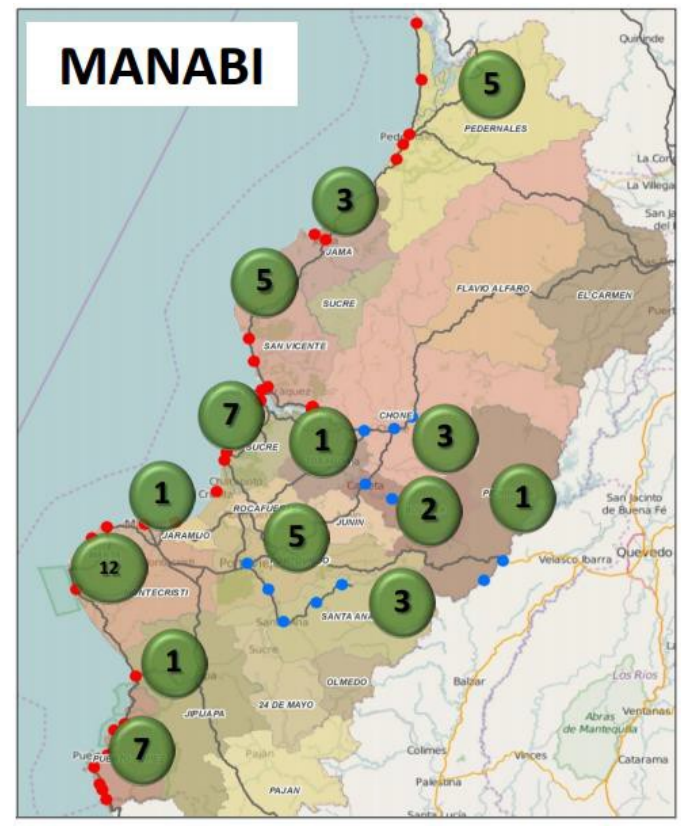

\section{Cantones}

\section{6 sirenas}

\begin{tabular}{|l|c|}
\hline \multicolumn{1}{|c|}{ CANTÓN } & SIRENAS \\
\hline BOLIVAR & 2 \\
\hline CHONE & 3 \\
\hline JAMA & 3 \\
\hline JARAMIJO & 1 \\
\hline JIPIJAPA & 1 \\
\hline MANTA & 12 \\
\hline PEDERNALES & 5 \\
\hline PICHINCHA & 1 \\
\hline PORTOVIEJO & 5 \\
\hline PUERTO LOPEZ & 7 \\
\hline SAN VICENTE & 5 \\
\hline SANTA ANA & 3 \\
\hline SUCRE & 7 \\
\hline TOSAGUA & 1 \\
\hline
\end{tabular}

44 sirenas tsunami

12 sirenas represas

Figura 2. Ubicación de alertas temprana para tsunamis y represas en Manabí Fuente: ECU 911, Ecuador, (2017) 


\section{Referencias Bibliográficas}

Alvarado, A. (2018). 16 de Abril de 2016: Lecciones Aprendidas recuperado de https://www.igepn.edu.ec/interactuamos-con-usted/1574-16-de-abril-de-2016-leccionesaprendidas-dra-alexandra-alvarado

Ander-Egg, E. (2010). Métodos y Técnicas de investigación social, Vol. III: Cómo organizar el trabajo de investigación. España: Lumen.

Banco Interamericano de Desarrollo, (2012). Indicadores de Riesgo de Desastre y de Gestión de $\begin{array}{llll}\text { Riesgos. } & \text { Ecuador. } & \text { Recuperado }\end{array}$ http://idbdocs.iadb.org/wsdocs/getdocument.aspx?docnum=35160022

Basabe, P., Almeida, E., Ramón, P., Zeas, R., \& Alvarez, L. (1996). Avance en la prevención de desastres naturales en la cuenca del río Paute, Ecuador. Bull. Inst. fr. études andines, 25(3), 443-458.

Bustos, J. D. (2010). Los movimientos telúricos en la ciudad de Cuenca, estrategias de comunicación no convencionales. Tesina previa obtención del título de Licenciado en Ciencias de la Comunicación. Universidad de Cuenca, Facultad de filosofía, letras y ciencias de la educación, Escuela de comunicación social, 14 de julio de 2010. Disponible en http://dspace.ucuenca.edu.ec/bitstream/123456789/1769/1/tc240.pdf

Caputo, M. G., Hardoy, J. E., \& Herzer, H. M. (1985). Desastres naturales y sociedad en América Latina. GEl: distribuido por EMECE Editores, Buenos Aires, AR.

Corral Muñoz, N. A. (2018). Efectos económicos provocados por el terremoto al turismo en Manta provincia de Manabí-Ecuador años 2016-2017 (Bachelor's thesis, Universidad de Guayaquil. Facultad de Ciencias Económicas).

Demoraes, F., \& D'ercole, R. (2001). Cartografía de riesgos y capacidades en el Ecuador. Mapa de amenazas, vulnerabilidad y capacidades en el Ecuador: Los desastres, un reto para el desarrollo. Coopi, Oxfam Internacional, SIISE. Quito, Ecuador.

ECU 911. (2017). Boletín informativo del Servicio Integrado de Seguridad. Ecuador. Disponible en http://www.ecu911.gob.ec/wp-content/uploads/2017/06/SATEcuador.pdf 
Estrada, R. B. y Farbiarz, F. J., (2005). El terremoto y posterior tsunami del 26 de diciembre de 2004 en indonesia. Dyna Revista de la Facultad de Minas de la Universidad Nacional de Colombia Vol. 72 No.145 Medellín (85 - 90) Enero - Abril 2005. ISSN 0012-7353

Geo Enciclopedia (2017). Cinturón de Fuego del Pacífico. Recuperado de https://www.geoenciclopedia.com/cinturon-de-fuego-del-pacifico/

Hernández Sampieri, R., Fernández Collado, C., \& Baptista Lucio, P. (2006). Metodología de la investigación (Vol. 3). México: McGraw-Hill.

Hernandez, Sampieri. R., Fernadez, C. C. y Baptista, L. M. (2014). Metodología de la investigación. México D F. México: Mc Graw Hill

Hernández-Sampieri, R. H., Collado, C. F., \& Lucio, P. B. (2012). Metodología de la Investigación. McGraw-Hill.

INEC, I. (2010). Censo de población y vivienda. Censo de Población y Vivienda.

Lavell, A. (1999). Gestión de riesgos ambientales urbanos. Red de Estudios Sociales en Prevención de Desastres en América Latina, www. desenredando. org.

López, M. C. (2013). Cronología de tsunamis en ecuador desde 1586 a 2012. La Técnica: Revista de las Agrociencias. e-ISSN 2477-8982, (11), 50-59.

Lugo Hubp, J., \& Inbar, M. (2002). Desastres naturales en América Latina (No. 363.34098 D441). Fondo de Cultura Económica.

Manchuel, K., Pontoise, B., Béthoux, N., Régnier, M., Font, Y., Sallares, V., ... \& Yépes, H. (2009). Sismicidad e implicaciones estructurales en el área de Esmeraldas (Norte de Ecuador): a partir de los experimentos Sublime y Esmeraldas. Geología y Geofísica Marina y Terrestre del Ecuador, 1st edn. Argudo \& Asociados, Guayaquil-Ecuador, 167179. 
Mothes, P. (Ed.). (1998). Actividad volcánica y pueblos precolombinos en el Ecuador. Editorial Abya Yala.

Ventura Velázquez, R. E., Menéndez López, J., Moreno Puebla, R. A., Gil Sánchez, R., Reyes Pérez, S., \& Cruz Minerva Turró, M. (2009). Los desastres como fenómenos diferentes a la guerra. Revista Cubana de Medicina Militar, 38(2), 0-0. 